\title{
Validation of inertial sensor to measure velocity of medicine balls
}

\author{
Kimitake Sato, Kevin M. Carroll, John P. Wagle, Henry M. Lang, Austin P. Smith, \\ John C. Abbott, Kaela M. Hierholzer, Michael H. Stone
}

Objectives: The purpose of this study was to examine the validity of a wireless device measuring velocity via inertial sensor medicine ball.

Design and Methods: Sixteen healthy adults volunteered in the study. Each participant performed a series of three static and countermovement (CM) medicine ball chest throws. All throws were performed using 8-lb and 12-lb medicine balls inlayed with a wirelessly transmitted accelerometer and gyroscope. Reflective markers were placed on both sides of medicine ball and data were collected using a three-dimensional (3D) motion analysis system as the criterion measure. Pearson correlations and paired samples t-tests were calculated to assess the accuracy of the medicine ball peak velocity to that of the 3D motion analysis. Additionally, intraclass correlation coefficients (ICC) were calculated within each device to determine reliability. The alpha level was set as $p \leq 0.05$.

Results: Pearson correlations indicated the medicine ball device to be relatively accurate with 3D motion analysis for static throws $(r=0.85-0.94)$ and CM throws $(r=0.62-0.89)$. There were no statistically significant differences between the two devices. ICC indicated trial-to-trial reliability of the medicine ball device to be acceptable ( $\mathrm{ICC}=0.74-0.98)$ compared to the $3 \mathrm{D}$ motion analysis ( $\mathrm{ICC}=0.67-0.98)$.

Conclusion: Overall, the study demonstrated that relatively accurate data may be obtained from an inertial sensor medicine ball, indicated from the strong and very strong correlations with 3D motion analysis. Additionally, similar ICC values between the medicine ball and 3D motion analysis suggest the device yields acceptable reliability.

(Journal of Trainology 2018;7:16-20)

Key words: velocity-based training $\mathbf{\square}$ athlete monitoring $\boldsymbol{\square}$ resistance training

\section{INTRODUCTION}

Velocity measurement in strength and conditioning has surged in popularity recently with the emergence of new technology for data collection..$^{1-3}$ While the concept of velocitybased training (VBT) has been around for years, ${ }^{4-6}$ these new technologies have made velocity measurement accessible to practitioners. Because of this accessibility, researchers have sought to explore the potential uses for VBT in practice. ${ }^{7-9}$ For example, VBT has been studied as a method of one-repetition maximum (1RM) prediction, ${ }^{7}$ fatigue identification, ${ }^{10}$ and perhaps most importantly, training load quantification and prescription $^{11,12}$. It has been established that a load-velocity relationship $(r=\sim 0.95)$ exists during progressive resistance training tests. ${ }^{7,11}$ However, VBT is typically only investigated for dumbbell ${ }^{13}$ or barbell ${ }^{11,14}$ exercises. Other common resistance training exercises, such as medicine ball variations, should also be explored if practitioners are interested in quantifying and prescribing resistance training loads.

Commercially available velocity measurement devices typically use one of two main hardware components; linear position transducers or accelerometers. Wireless accelerometry devices sold for the measurement of velocity in resistance training use Bluetooth and algorithm-based data acquisition to estimate velocity from acceleration-time data. Companies develop their own proprietary algorithms, there is considerable caution for differences in calculations and resultant information. Therefore, it is vital for researchers to determine the accuracy and consistency of new devices before they are used in an applied VBT setting. Many of these devices have been tested for validity and reliability in dumbbell and barbell exercises using gold standard criterions. ${ }^{2,13}$ However, medicine ball exercises present a unique challenge for technology manufacturers because of the versatile nature of many medicine ball exercise variations.

The purpose of this study was to examine the validity of a commercially available device which measures velocity via an inertial sensor medicine ball. The study was to determine the validity of the wireless device (medicine ball) with a gold standard instrument (3D motion analysis) using two chest throw exercises. Additionally, the external reliability of the device was assessed from the multiple trials each participant completed during the testing protocol.

The importance of this study lies in the potential ability to quantify medicine ball exercises using velocity measurement.

Received February 15, 2018; accepted June 16, 2018

From the Department of Sport, Exercise, Recreation, and Kinesiology, Center of Excellence for Sport Science and Coach Education, East Tennessee State University, Johnson City, TN, USA (K.S., K.M.C., J.P.W., H.M.L., A.P.S., J.C.A., K.M.H., M.H.S.)

Communicated by Takashi Abe, PhD

Correspondence to Dr. Kimitake Sato, Department of Sport, Exercise, Recreation, and Kinesiology, Campus box 70671, East Tennessee State University, Johnson City, TN 37614, USA. Email: satok1@etsu.edu 
While dumbbell and barbell exercises can be measured accurately with already available devices, the ability to quantify the medicine ball exercises has not been thoroughly examined yet. Monitoring these data could be beneficial for several important factors; 1) to compare the novice level athletes' values with higher level athletes, 2) to be able to monitor the data over time on an individual basis, and 3) to investigate how physical adaptation over time from novice level athletes to be able to throw medicine ball faster.

\section{Participants}

\section{METHODS}

All recruited participants $(\mathrm{N}=16)$ were healthy, active adults who had experience with medicine ball throw exercises, as they were all regularly trained individuals. The eligibility criteria included: no injuries inhibiting their ability to perform medicine ball throws, 18 years of age or older, engaged in resistance training. Study information was provided to all potential participants verbally prior to participation. Each participant read and signed a written informed consent that was approved by University Internal Review Board.

\section{Procedures}

After the participants completed their own self-selected static and dynamic warm-up, they performed a series of three static and countermovement (CM) medicine ball chest throws. All throws were performed using 8-1b and 12-lb inertial sensor medicine balls (Assess2Perform, Steamboat Springs, CO, USA). The inertial sensor was located at the center of each device. Warm-up trials at $50 \%$ and $75 \%$ of perceived maximum effort were done prior to the first recorded static and CM chest throws to have familiarization. For the static chest throw, participants bent elbows to a $90^{\circ}$ angle with an athletic standing position. Once the proper position was reached, a "3-2-1 throw" command was given to initiate the throws. For the CM chest throw, participants began from an extended arm position directly in front of the chest and were asked to throw the medicine ball using a self-selected CM depth. The throwing test was completed when data were collected from three properlyexecuted static and CM chest throws using both 8-1b and 12-1b medicine balls. A total of 12 throws from each participant were considered for analysis. It is important to note that since this was a validation study for device vs. device, details such as depth of CM and individual strength level were not regulated. Also, sex, height, and weight of participant's variability was not a part of study criteria as those should not affect the control of validation process.

\section{Data Processing}

Reflective markers were placed on the center of medicine ball (left and right sides) to track the markers. The company reported that the sampling frequency of all medicine balls was $100 \mathrm{~Hz}$. Determined through a series of pilot tests, a 3D motion analysis system with six cameras (Vicon Nexsus 1.8.11, Centennial, CO USA) synchronized to sample at $200 \mathrm{~Hz}$ to ensure the peak velocity occurred as an instantaneous point of release from hands to match up with medicine ball's peak velocity. The reflective markers were also placed on dorsal side of hands (left and right) to track hands motion. This was to detect hand markers' moving coordinates to identify the medicine ball takes off from hands to capture the instantaneous point of release (i.e., medicine ball being airborne). Calibration of the motion analysis system was done prior to each data collection session at less than $0.5 \%$ error in the image volume to minimize the error of analysis. Velocity from the motion analysis system was processed using 3D coordinates of each marker set. Three-dimensional (X, Y, \& Z) movement of reflective markers were considered to calculate resultant velocity from the motion analysis system to obtain the peak velocity. A filtering process was used for the 3D motional analysis data using a low pass fourth-order Butterworth filter with cutoff frequencies of $15 \mathrm{~Hz}$. The medicine ball velocity was displayed using the company's smartphone application (Assess2Perform, Steamboat springs, CO USA), and recorded manually into spreadsheet.

\section{Data and Statistical Analysis:}

For validation analysis, there were two different ways to analyze the data. First, the study considered all trials for analysis. A grand total of 192 throws (16 participants with 12 throws) for static and CM chest throws in each load condition (8-lb \& 12-lb). It was necessary to take all successful data into consideration for a validity purpose to examine the accuracy of medicine ball velocity. Second, averaged data from three throws from each condition were also considered for correlation with the corresponding data from 3D motion analysis software (a total of 64 data). The specific variable that was considered in this study was peak velocity.

A series of Pearson correlation coefficient analyses were run to determine the relationship between the two devices (medicine ball and 3D motion analysis) for both all trial and averaged trial velocity data. A series of paired-samples t-tests were also performed to identify the differences when comparing velocity derived from the medicine ball to the $3 \mathrm{D}$ motion analysis system. The alpha level was set as $p \leq 0.05$. Pearson's $r$ values were interpreted with magnitude thresholds previously established by Hopkins: $r=0-0.1$ is trivial, $r=0.1-0.3$ is small, $r=0.3-0.5$ is moderate, $r=0.5-0.7$ is large, $r=0.7-0.9$ is very large, and $r=0.9-1.0$ is nearly perfect. ${ }^{15}$

Although reliability was a secondary purpose, each trial's data were analyzed for consistency identification within each condition. Although this analysis was not fully controlled in this study, it was necessary to identify whether that consistency of data output was similar between the medicine ball and 3D motion analysis system to ensure its measurement reliability. All three throws from each conditioning were used to calculate within-condition of each participant intra-class correlation coefficient (ICC) reliability analysis.

\section{RESULTS}

Descriptive data from the testing is represented in Table 1. When all trial data were analyzed simultaneously, large to nearly perfect correlations were observed for static throw with 8-lb $(r=0.881)$, with 12-lb $(r=0.918)$, and CM throw with 
Table 1 Demographic data on all velocity data $(\mathrm{m} / \mathrm{s})$ with Cohen's d effect size.

\begin{tabular}{rcccccc}
\hline & \multicolumn{2}{c}{ Medicine Ball } & \multicolumn{2}{c}{ 3D motion analysis } & \multicolumn{2}{c}{ Cohen's d Effect size } \\
& $8 \mathrm{lb}$ & $12 \mathrm{lb}$ & $8 \mathrm{~b}$ & $12 \mathrm{lb}$ & $8 \mathrm{~b}$ & $12 \mathrm{lb}$ \\
\hline Static throw & $3.94 \pm 0.506$ & $3.92 \pm 0.644$ & $4.16 \pm 0.828$ & $3.96 \pm 0.905$ & 0.32 & 0.05 \\
CM throw & $4.33 \pm 0.634$ & $4.15 \pm 0.715$ & $4.61 \pm 0.910$ & $4.43 \pm 0.950$ & 0.36 & 0.33 \\
\hline
\end{tabular}
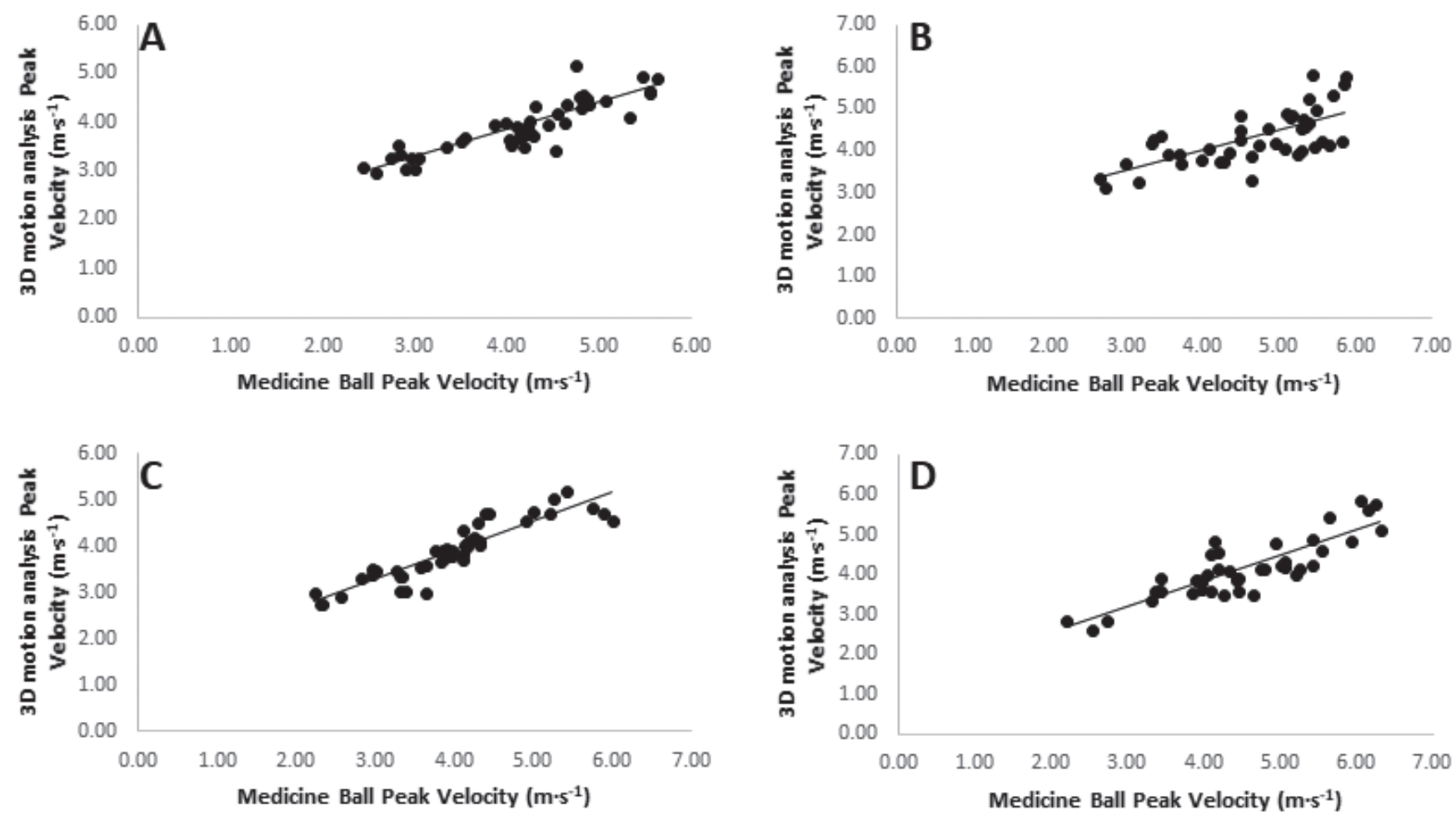

Figure 1 Peak velocity obtained from the 3D motion analysis system is represented on the $y$-axis while the peak velocity obtained from the medicine ball is represented on the $\mathrm{x}$-axis for: A) static 8-Ib $(r=0.921)$, B) counter-movement 8-Ib $((r=0.693)$, C) static 12-b ( $r=0.921)$, and D) counter-movement 12-lb $((r=0.877)$ throws.

8-lb $(r=0.675)$, and with 12-lb $(r=0.854)$. Scatter plots of these data are represented in Figure 1. When trials were averaged, strong correlations for peak velocity were observed between the devices using static throw with 8-lb $(r=.921)$, with 12-lb $(r=.921), \mathrm{CM}$ throw with $8-\mathrm{lb}(r=.693)$, and with $12-\mathrm{lb}(r=.877)$.

Data from each device were also compared using pairedsamples t-tests to determine the absolute differences in velocity data. All paired samples t-tests showed no statistically significance differences between peak velocities from both the medicine ball and the 3D motion analysis system (static throw $8 \mathrm{lb}(t(15)=2.126, p=0.051)$, static throw $12 \mathrm{lb}(t(15)=0.480$, $p=0.638), \mathrm{CM}$ throw $8 \mathrm{lb}(t(15)=1.695, p=0.112), \mathrm{CM}$ throw $12 \mathrm{lb}(t(15)=1.278, p=0.303)$. Further, Bland-Altman analysis (Figure 2) indicated a slight bias towards 3D motion analysis at lower PV and towards the medicine ball at higher velocities. Both ICC and CV indicated high reliability and low variations, obtained from the medicine ball were similar in magnitude to those obtained from the 3D motion analysis (Table 2).

\section{DISCUSSION}

The purpose of the study was to measure the accuracy of the values obtained using a wireless inertial sensor medicine ball at two different weights compared to a $3 \mathrm{D}$ motion analysis system. Overall, the results demonstrated that the accelerometer accurately captured the peak velocity, indicated by its strong correlation with 3D motion analysis system.

It is encouraging to capture strong correlation in most conditions as algorithm-based devices have not always shown high accuracy previously, especially for instantaneous data such as peak velocity. ${ }^{2}$ The relatively high agreement between the device and SD motion analysis is encouraging for its use in practical settings and provides insight into how the device actually measures velocity output. An interesting finding comes from the Bland-Altman analysis. It seems that there is a slight bias towards the $3 \mathrm{D}$ motion analysis at lower peak velocities and towards the medicine ball at higher velocities (Figure 2). This result was likely due to differences in sampling rate between the medicine ball and the 3D motion analy- 

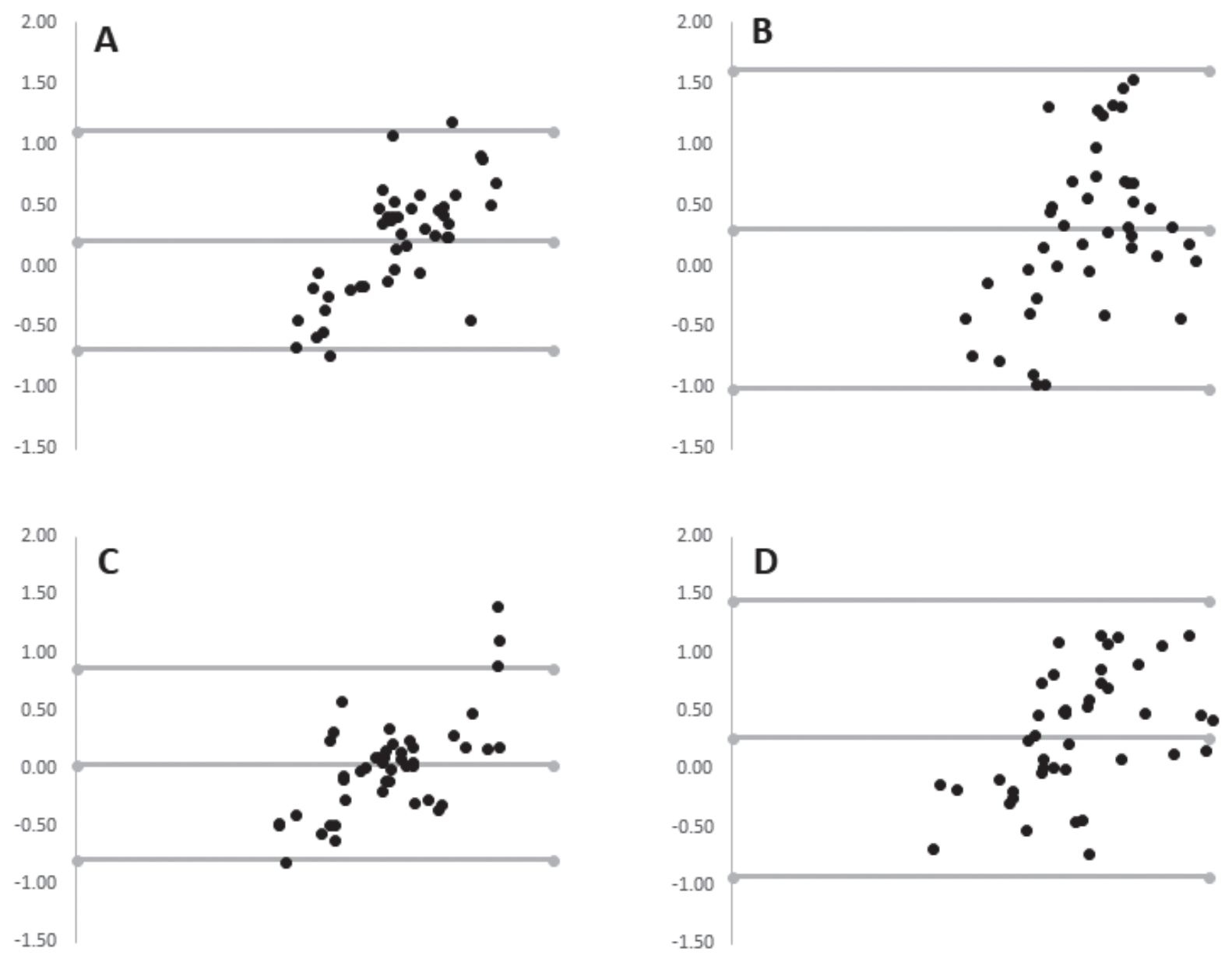

Figure 2 Bland-Altman plots for medicine ball and 3D motion analysis data for: A) static 8-Ib, B) counter-movement 8-Ib, C) static 12-b, and D) counter-movement 12-lb throws.

Table 2 Intra-session coefficient correlation and coefficient of variation for both measures.

\begin{tabular}{cccccc}
\hline & \multicolumn{2}{c}{ Medicine ball } & \multicolumn{2}{c}{ 3D motion capture system } \\
\hline \multirow{2}{*}{ Static throw } & & $8 \mathrm{lb}$ & $12 \mathrm{lb}$ & $8 \mathrm{lb}$ & $12 \mathrm{lb}$ \\
\cline { 2 - 6 } & $\mathrm{ICC}$ & $.79-.89$ & $.89-.97$ & $.67-.76$ & $.84-.96$ \\
\cline { 2 - 6 } $\mathrm{CV}$ & $0.00-12.89 \%$ & $1.19-11.65 \%$ & $0.35-13.21 \%$ & $0.43-9.18 \%$ \\
\hline \multirow{2}{*}{ CM throw } & $\mathrm{ICC}$ & $.86-.91$ & $.74-.91$ & $.85-.98$ & $.91-.97$ \\
\cline { 2 - 6 } & $\mathrm{CV}$ & $1.47-10.33 \%$ & $0.89-10.61 \%$ & $0.46-8.70 \%$ & $0.13-10.24 \%$ \\
\hline
\end{tabular}

sis. However, future research might consider examining reliability in typically high vs. low velocity medicine ball exercises.

The traditional means of measuring medicine ball throw output have relied on distance travelled. Having the ability to measure the peak velocity of medicine ball exercises would be useful for practitioners, as this is mostly independent of selfselected trajectory and technique and allow for more objective comparisons in athlete monitoring. For example, distance of throw is determined by 3 factors; velocity at take-off, height of release, and angle of release. In developing effective athlete monitoring practices, sport scientists should seek measures that control for as many factors as possible in order to make objective comparisons between and within athletes. Furthermore, if physical outputs are the major interest for coaches, peak velocity would be an efficacious variable to compare different levels of athletes in medicine ball exercises, or use the data for long term monitoring.

Practically speaking, it is not easy to have identical throwing mechanics at every attempt, but it is necessary to have "intent for maximal effort" when implementing monitoring tests such as medicine ball throws. Therefore, peak velocity may be the most appropriate measure to select, as it measures the result of maximal output. As this investigation's data support the accu- 
racy of the device with adequate reliability, now the speculation can turn to explore the relationship between medicine ball peak velocity and other common assessments of physical ability, such as sprinting or jumping. This may lead to not only exploring the efficacy of tracking medicine ball peak velocity as a strategy of athlete monitoring, but also develop best practices in training. For example, sport scientists may consider the drop-off percentage with different weights of medicine balls during training, using a similar approach to velocity measurement during conventional resistance training exercises. ${ }^{10}$

This type of wireless device has become popular in sport technology, and companies often compete the usefulness for the accuracy and reliability of their devices, as any device's utility depends on these two rudimentary factors. In the present study, data was relatively similar between the medicine ball and $3 \mathrm{D}$ motion analysis system. However, it is important to remind the reader that the $3 \mathrm{D}$ motion analysis was taken at double of sampling frequency than that of the medicine ball. This was a necessary procedure to assure the capture of instantaneous peak velocity during throws. As the medicine ball was sampled at $100 \mathrm{~Hz}$, it is reasonable to question as to whether the current sampling rate is fast enough to capture high output rate medicine ball throws. One evidence from current data is 8-lb CM chest throws as they are the fastest peak velocity, showed widest margins of peak velocity from 3D motion analysis system. This indicates that the CM action plus light intensity may cause the small error of peak velocity calculation from their algorithm. Additional consideration can be given to the results of the Bland-Altman analysis which also revealed a slight velocity-dependent bias on agreement between the devices. This could be corrected from faster sampling frequency and fine-tuned algorithm.

As the present study did not aim to examine other throws, future investigation should examine the accuracy of throws with different throwing velocity. It is noticeable that the $\mathrm{CV}$ values on all throws ranging up to above $10 \%$. This is not necessarily coming from device's error since both medicine ball and $3 \mathrm{D}$ motion analysis system showed relatively the same range. This may be due to participants' technique to cause its variation between throws. If that is the case, the medicine ball may serve as a training tool for athletes to minimize the throwing technique variation. This approach of technique analysis need further investigation as a medicine ball velocity measure could serve as a technical training tool.

Overall, the study demonstrated that relatively accurate data obtained from an inertial sensor medicine ball, indicated from the large and very large correlations with 3D motion analysis. Similar ICC and CV values between the medicine ball and 3D motion analysis suggest the device yields acceptable reliability.

\section{REFERENCES}

1. Balsalobre-Fernandez C, Marchante D, Baz-Valle E, Alonso-Molero I, Jimenez SL, Munoz-Lopez M. Analysis of Wearable and SmartphoneBased Technologies for the Measurement of Barbell Velocity in Different Resistance Training Exercises. Front Physiol 2017;8:649.

2. Banyard HG, Nosaka K, Sato K, Haff GG. Validity of Various Methods for Determining Velocity, Force, and Power in the Back Squat. Int J Sports Physiol Perform 2017;12:1170-1176.

3. Cronin J, McNair P, Marshall R. Is velocity-specific strength training important in improving functional performance? J Sports Med Phys Fitnes 2002;42:267-273.

4. Kanehisa H, Miyashita M. Specificity of velocity in strength training. Eur J Appl Physiol Occup Physiol 1983;52:104-106.

5. Pereira MI, Gomes PS. Movement velocity in resistance training. Sports Med 2003;33:427-438.

6. Izquierdo M, Gonzalez-Badillo JJ, Hakkinen K, et al. Effect of loading on unintentional lifting velocity declines during single sets of repetitions to failure during upper and lower extremity muscle actions. Int J Sports Med 2006;27:718-724.

7. Judovtseff B, Harris NK, Crielaard J, Cronin JB. Using the load-velocity relationship for 1RM prediction. J Strength Cond Res 2011;25:267-270.

8. Jovanovic M, Flanagan EP. Researched applications of velocity based strength training. J Aust Strength Cond 2014;22:58-69.

9. Gonzalez-Badillo JJ, Pareja-Blanco F, Rodriguez-Rosell D, Abad-Herencia JL, Del Ojo-Lopez JJ, Sanchez-Medina L. Effects of velocity-based resistance training on young soccer players of different ages. J Strength Cond Res 2015;29:1329-1338.

10. Sanchez-Medina L, Gonzalez-Badillo JJ. Velocity loss as an indicator of neuromuscular fatigue during resistance training. Med Sci Sports Exerc 2011;43:1725-1734.

11. Gonzalez-Badillo JJ, Sanchez-Medina L. Movement velocity as a measure of loading intensity in resistance training. Int J Sports Med 2010;31:347352.

12. Banyard HG, Nosaka K, Haff GG. Reliability and Validity of the LoadVelocity Relationship to Predict the 1RM Back Squat. J Strength Cond Res 2017;31:1897-1904.

13. Sato K, Beckham GK, Carroll KM, Bazyler CD, Sha Z, Haff GG. Validity of wireless device measuring velocity of resistance exercises. J Trainol 2015;4:15-18.

14. Sakamoto A, Sinclair PJ. Effect of movement velocity on the relationship between training load and the number of repetitions of bench press. $J$ Strength Cond Res 2006;20:523-527.

15. Hopkins WG, Marshall SW, Batterham AM, Hanin J. Progressive statistics for studies in sports medicine and exercise science. Med Sci Sports Exerc 2009;41:3-13. 